\title{
A Minimal Touch Approach for Optimizing Energy Efficiency in Pick-and-Place Manipulators
}

\author{
Marcello Pellicciari, Giovanni Berselli, Francesco Leali, Alberto Vergnano
}

\begin{abstract}
The interest in novel engineering methods and tools for optimizing the energy consumption in robotic systems is currently increasing. In particular, from an industry point of view, it is desirable to develop energy saving strategies applicable also to established manufacturing systems, being liable of small possibilities for adjustments. Within this scenario, an engineering method is reported for reducing the total energy consumption of pick-and-place manipulators for given end-effector trajectory. Firstly, an electromechanical model of parallel/serial manipulators is derived. Then, an energy-optimal trajectory is calculated, by means of time scaling, starting from a pre-scheduled trajectory performed at maximum speed (i.e. compatible with actuators limitations). A simulation case study finally shows the effectiveness of the proposed procedure.

Index Terms-Pick-and-place manipulators, energy efficiency
\end{abstract}

\section{INTRODUCTION}

The development of energy efficient mechatronic systems is currently changing standard paradigms in the design and control of robotic devices. Many approaches for Energy Loss (EL) minimization are found in different research fields, such as introduction of renewable energy sources [1], device design optimization [2]-[4], efficient equiplment selection [5], [6], path planning [7], [8], up to the optimization of the overall production system [9]. Nonetheless, these strategies can be applied on different time frames. In fact, renewable energies will find a global impact in a mid/long term goal whereas new energy-efficient equipment (e.g. lightweight robots [10]) might not be readily applied to real systems due to market constraints, as costs and/or production rates.

Still, most energy saving methods described in literature rely on equipment selection or replacement, plant modification or path re-planning. Hence, they involve considerable system modifications, which can be adopted only in a new plant design process. In case of an established system optimization, an effective method must trade off between the involved fixed costs for equipment investments and the reduction of the variable cost for energy. Also, these system are usually technologically optimized and liable of small possibilities for adjustments due to quality issues. Therefore, when a system is in this mature lifecycle phase, it is surely desirable to reduce the EL while involving little or no further investments. In particular, a large possibility of improvement concerns existing robotic manufacturing systems which are far from their lifecycle end and, therefore, are kept productive without any substantial modification.

Marcello Pellicciari, Giovanni Berselli, Francesco Leali, Alberto Vergnano are Assistant Professors at DIMEC, Mechanical Eng. Dept., University of Modena and Reggio Emilia, Via Vignolese 905, 41125 Modena, Italy (email: name.surname@unimore.it.)

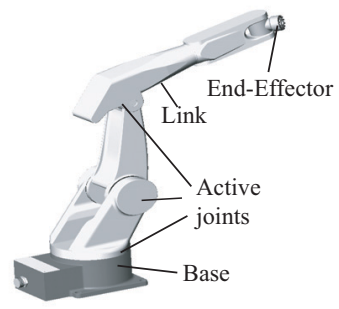

(a) Six d.o.f. serial robot

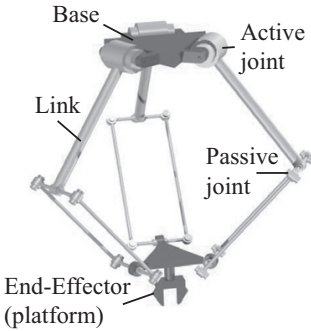

(b) Three d.o.f. parallel robot
Fig. 1. Industrial robots for pick-and-place operations

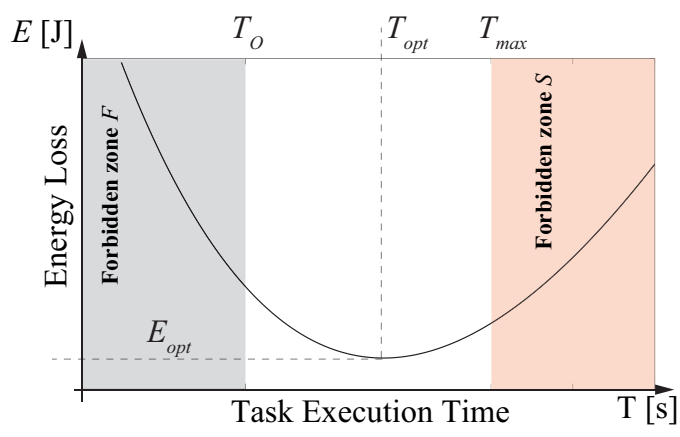

Fig. 2. Energy Consumption as a function of Task Execution Time (TET).

Within this scenario, the focus of this paper is to present a novel method for EL minimization in robotic manufacturing cells by means of a minimal touch approach. Differently from the aforementioned design methods aiming at minimizing EL, the electromechanical system characteristics are assumed as given (i.e. no additional costs are expected), the only parameter to be varied being the Task Execution Time (TET), $T$. In fact, in many practical applications (e.g. manipulation or spot welding operations), the TET can be assessed in order to obtain energy-optimal scheduling, compatible with the cell production rates [9], [11].

The concept is well described considering a pick-and-place operation performed by either a 6 degrees of freedom (d.o.f.) industrial robot (Fig. 1(a)) or a common 3 d.o.f. Parallel Kinematic Machine (PKM) (Fig. 1(b)). The overall system EL during the operation is qualitatively depicted in Fig. 2 as a function of TET. The energy needed to perform the operation increases, with respect to a global optimum $T_{o p t}$, for both low and high TET (i.e. fast or slow speed respectively). On the other hand, many scheduling optimization methods (e.g. [12], [13]), assume that the robot operates at its maximum speed, whenever allowed by the scheduling constraints, and stands 
still otherwise (idle times). However, such task planning strategy may be energetically detrimental, leading to high power consuming accelerations and longer idle times (where energy is wasted to counteract gravitational loads). Note that, in any case, very low TET cannot be achieved due to torque limitations of the actuation system (forbidden zone $F$ in Fig. 2 ), whereas very high TET are considered forbidden as long as they might negatively affect the production rate (forbidden zone $S$ in Fig. 2).

In this context, according to the mentioned minimal touch approach, this paper focuses on EL minimization of pick-and-place operations for given end-effector trajectories. Firstly, an electromechanical model of common parallel/serial manipulators is derived. Then, the energy consumption in function of the task execution time is calculated, by means of time scaling [14], starting from a pre-scheduled trajectory performed at maximum speed.

\section{Electro-MECHANiCAL MODELING OF INDUSTRIAL ROBOTS}

As previously hypothesized, the present method is conceived to optimize a system without new hardware investments or invasive operation modifications. To that purpose the method assumes the system hardware parameters as assigned. Also, the robot end-effector trajectory is given, and no changes can be introduced for any previously defined operation. Similarly to other approaches (see e.g. [4]), the robot electromechanical model consists of the contribution of mechanism rigid-body dynamics, electric servo motors and eventual speed reducers (including gear friction).

\section{A. Background on Serial Manipulators Dynamic Model}

A typical $n$-link serial manipulator (an open kinematic chain), as shown in Fig. 1(a), is actuated by one motor for each single joint. It is supposed that each motor is located on the robot link preceding the driven link. The manipulator joint space dynamic model [15] can be written as:

$$
\boldsymbol{\tau}_{r}=\mathbf{M}_{r}\left(\mathbf{q}_{r}\right) \ddot{\mathbf{q}}_{r}+\mathbf{V}_{r}\left(\mathbf{q}_{r}, \dot{\mathbf{q}}_{r}\right)+\mathbf{G}_{r}\left(\mathbf{q}_{r}\right)+\mathbf{J}_{r}{ }^{T}\left(\mathbf{q}_{r}\right) \mathbf{h}
$$

where $\mathbf{q}_{r}(t), \dot{\mathbf{q}}_{r}(t)$, and $\ddot{\mathbf{q}}_{r}(t) \in \mathbb{R}^{n}$ represent, respectively, the angular positions, velocities, and accelerations of the robot joints, as function of time $t . \mathbf{M}_{r}\left(\mathbf{q}_{r}\right) \in \mathbb{R}^{n \times n}$ is the symmetric joint-space inertia matrix, $\mathbf{V}_{r}\left(\mathbf{q}_{r}, \dot{\mathbf{q}}_{r}\right) \in \mathbb{R}^{n}$ describes the Coriolis/centripetal torques, $\mathbf{G}_{r}\left(\mathbf{q}_{r}\right) \in \mathbb{R}^{n}$ is the gravity loading, $\boldsymbol{\tau}_{r} \in \mathbb{R}^{n}$ is the vector of generalized forces associated to $\mathbf{q}_{r}$, $\mathbf{h} \in \mathbb{R}^{6}$ is the wrench exerted by the environment on the endeffector, $\mathbf{J}_{r}\left(\mathbf{q}_{r}\right) \in \mathbb{R}^{n \times n}$ is the manipulator jacobian matrix. Friction forces (mainly due to gear reducers) will be modeled within the actuation subsystem (Sec. II.C).

The Coriolis/centripetal vector $\mathbf{V}_{r}$ can be written in different forms. For instance, a widely extended tensor representation is based on Christoffel symbols of the first kind [15]. Nonetheless, for the purpose of the following sections, it is useful to recall a tensorial notation introduced by [16]:

$$
\begin{aligned}
\mathbf{V}_{r}\left(\mathbf{q}_{r}, \dot{\mathbf{q}}_{r}\right) & =\left[\begin{array}{c}
\dot{\mathbf{q}}_{r}^{T} \mathbf{V}_{1 r}\left(\mathbf{q}_{r}\right) \dot{\mathbf{q}}_{r} \\
\vdots \\
\dot{\mathbf{q}}_{r}^{T} \mathbf{V}_{1 r}\left(\mathbf{q}_{r}\right) \dot{\mathbf{q}}_{r}
\end{array}\right]=\left(\mathbf{I}_{n} \otimes \dot{\mathbf{q}}_{r}^{T}\right)\left[\begin{array}{c}
\mathbf{V}_{1 r}\left(\mathbf{q}_{r}\right) \\
\vdots \\
\mathbf{V}_{n r}\left(\mathbf{q}_{r}\right)
\end{array}\right]_{(2)} \dot{\mathbf{q}}_{r} \\
& \equiv\left(\mathbf{I}_{n} \otimes \dot{\mathbf{q}}_{r}^{T}\right) \overline{\mathbf{V}}_{r}\left(\mathbf{q}_{r}\right) \dot{\mathbf{q}}_{r}
\end{aligned}
$$

where $\mathbf{I}_{n} \in \mathbb{R}^{n \times n}$ denotes the identity matrix, the symbol $\otimes$ denotes the Kronecker product [16] and an appropriate definition of $\mathbf{V}_{i r}\left(\mathbf{q}_{r}\right) \in \mathbb{R}^{n \times n}(i=1, n)$ can be found in [15].

In the case of pick-and-place operations, the vector $\mathbf{h}$ is simply given by the equations of motion of the handled object:

$$
\mathbf{h}=\underbrace{\left[\begin{array}{c}
m \ddot{\mathbf{p}} \\
\mathfrak{\omega} \dot{\omega} \times \mathfrak{I} \omega
\end{array}\right]}_{\mathbf{h}_{I}}+\underbrace{\left[\begin{array}{c}
m \mathbf{g} \\
\mathbf{0}
\end{array}\right]}_{\mathbf{h}_{g}}
$$

where $m$ is the mass of the object, $\mathbf{g} \in \mathbb{R}^{3}$ is the gravity vector (in appropriate units and direction), $\mathfrak{I} \in \mathbb{R}^{3 \times 3}$ is the inertial matrix of the object, and $\mathbf{0} \in \mathbb{R}^{3}$ is the null vector. The object position, $\mathbf{p}(t) \in \mathbb{R}^{3}$, and angular velocity, $\omega(t) \in \mathbb{R}^{3}$, are expressed with respect to a fixed reference Cartesian frame and can be written in compact form as $\mathbf{v}=[\mathbf{p}(t) \mid \omega(t)]^{T} \in \mathbb{R}^{6}$. For the purpose of the following sections, in Eq. 3, it is convenient to isolate the inertial, $\mathbf{h}_{I}$, and gravitational, $\mathbf{h}_{g}$, contributions of the external wrench $\mathbf{h}$.

\section{B. Background on Parallel Manipulators Dynamic Model}

A typical PKM (a closed kinematic chain) is depicted in Fig. 1(b). In order to derive the PKM dynamic model, a possible approach is to consider a reduced system, i.e. a tree topology mechanism obtained by cutting the loops in a closed chain mechanism (see e.g. [17]). The dynamics of the reduced system (an open kinematic chain) can be described by an equation of the same form of Eq. 1 where, however, not all the pairs are actuated. The original (closed) and the reduced system are then assumed to experience the same external forces and to undergo the same motions.

In particular, let the closed chain consist of a total of $k$ one d.o.f. joints, $n$ of which are actuated. Let $\mathbf{q}_{a} \in \mathbb{R}^{n}, \mathbf{q}_{r} \in \mathbb{R}^{m}$ (where $n<m$ ) denote a set of independent generalized coordinates for the closed chain and its corresponding reduced system, respectively. In the same manner, let $\tau_{a} \in \mathbb{R}^{n}$ and $\boldsymbol{\tau}_{r} \in \mathbb{R}^{m}$ denote the external generalized forces of the original and reduced systems, respectively. In addition, let the joint vectors of the reduced system be partitioned as $\mathbf{q}_{r}=\left[\mathbf{q}_{p} \mid \mathbf{q}_{a}\right]^{T}$, where $\mathbf{q}_{a}$ is the actuated joint vector whereas $\mathbf{q}_{p} \in \mathbb{R}^{k-n}$ is the passive joint vector. The corresponding torque vector can be ordered and partitioned as $\boldsymbol{\tau}_{r}=\left[\boldsymbol{\tau}_{p} \mid \boldsymbol{\tau}_{a}\right]^{T}$, where $\tau_{a}$ includes actuator and friction torques (active joints), whereas $\tau_{p} \in \mathbb{R}^{k-n}$ includes friction contributions only. On the other hand, friction forces on passive joints, mainly due to sliding pairs, can be reasonably neglected with respect to friction forces within the actuator gear reducers. Therefore, $\boldsymbol{\tau}_{p}=\mathbf{0}$. Then, away from actuator singularity and imposing 


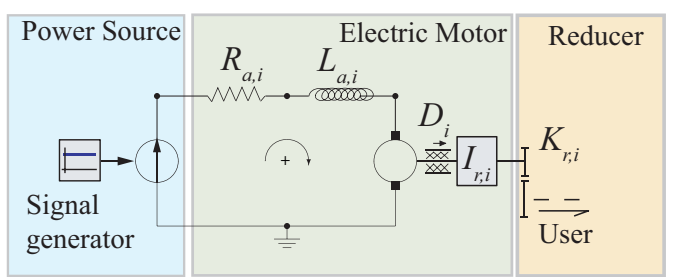

Fig. 3. Simplified electromechanical model of servo electric motor and speed reducer.

equivalent motions of the original and reduced system, $\mathbf{q}_{r}$ is functionally dependent on $\mathbf{q}_{a}$, as

$$
\begin{aligned}
\mathbf{q}_{r} & =\mathbf{q}_{r}\left(\mathbf{q}_{a}\right)=\left[\begin{array}{c}
\mathbf{q}_{a} \\
\mathbf{q}_{p}\left(\mathbf{q}_{a}\right)
\end{array}\right] \\
\dot{\mathbf{q}}_{r} & =\Phi \dot{\mathbf{q}}_{a} \\
\ddot{\mathbf{q}}_{r} & =\Phi \dot{\mathbf{q}}_{a}+\Phi \ddot{\mathbf{q}}_{a}
\end{aligned}
$$

The matrix $\Phi \in \mathbb{R}^{m \times n}$ is the generalized actuating force transformation [17] defined as:

$$
\begin{gathered}
\Phi\left(\mathbf{q}_{a}\right)=\frac{\partial \mathbf{q}_{r}}{\partial \mathbf{q}_{a}}=\left[\begin{array}{c}
\frac{\partial \mathbf{q}_{p}}{\partial \mathbf{q}_{a}} \\
\mathbf{I}_{n}
\end{array}\right] \\
\dot{\Phi}\left(\mathbf{q}_{a}, \dot{\mathbf{q}}_{a}\right)=\frac{d}{d t} \frac{\partial \mathbf{q}_{r}}{\partial \mathbf{q}_{a}}=\left[\begin{array}{c}
\frac{d}{d t} \frac{\partial \mathbf{q}_{p}}{\partial \mathbf{q}_{a}} \\
\mathbf{0}
\end{array}\right]
\end{gathered}
$$

In the same way, $\tau_{r}$ is functionally dependent on $\tau_{a}$, as

$$
\tau_{a}=\Phi^{T} \tau_{r}
$$

By applying Eqs.6 and 4 to the reduced system dynamic model in Eqs. 1 and 2, the following expression is found:

$$
\begin{aligned}
\tau_{a}= & \Phi^{T}\left[\mathbf{M}_{r}\left(\mathbf{q}_{r}\left(\mathbf{q}_{a}\right)\right)\left(\dot{\Phi} \dot{\mathbf{q}}_{a}+\Phi \ddot{\mathbf{q}}_{a}\right)+\right. \\
& +\left(\mathbf{I}_{n} \otimes\left(\Phi \dot{\mathbf{q}}_{a}\right)^{T}\right) \overline{\mathbf{V}}_{r}\left(\mathbf{q}_{r}\left(\mathbf{q}_{a}\right)\right) \Phi \dot{\mathbf{q}}_{a}+ \\
& \left.+\mathbf{G}_{r}\left(\mathbf{q}_{r}\left(\mathbf{q}_{a}\right)\right)+\mathbf{J}_{r}^{T}\left(\mathbf{q}_{r}\left(\mathbf{q}_{a}\right)\right) \mathbf{h}\right]
\end{aligned}
$$

Equation 7 can be written as:

$$
\begin{aligned}
\boldsymbol{\tau}_{a}= & \mathbf{M}_{1}\left(\mathbf{q}_{a}\right) \ddot{\mathbf{q}}_{a}+\mathbf{M}_{2}\left(\mathbf{q}_{a}\right) \dot{\Phi} \dot{\mathbf{q}}_{a}+\left(\mathbf{I}_{n} \otimes \dot{\mathbf{q}}_{a}^{T}\right) \overline{\mathbf{V}}\left(\mathbf{q}_{a}\right) \dot{\mathbf{q}}_{a}+ \\
& +\mathbf{G}\left(\mathbf{q}_{a}\right)+\mathbf{J}^{T}\left(\mathbf{q}_{a}\right) \mathbf{h}
\end{aligned}
$$

where:

$$
\begin{array}{rrr}
\mathbf{M}_{1}=\Phi^{T} \mathbf{M}_{r} \Phi & \mathbf{M}_{2}=\Phi^{T} \mathbf{M}_{r} \Phi \\
\mathbf{G}=\Phi^{T} \mathbf{G}_{r} & \mathbf{J}=\mathbf{J}_{r} \Phi \\
\overline{\mathbf{V}}=\left[\begin{array}{c}
\Phi^{T} \mathbf{V}_{1 r}\left(\mathbf{q}_{r}\right) \Phi \\
\vdots \\
\Phi^{T} \mathbf{V}_{n r}\left(\mathbf{q}_{r}\right) \Phi
\end{array}\right]
\end{array}
$$

Equation 8 describes parallel manipulator dynamics away from actuator singularity. The same equation reduces to Eqs. 1 and 2 whenever $\tau_{r} \equiv \boldsymbol{\tau}_{a}$ (i.e. $\Phi=\mathbf{I}_{n} \Rightarrow \dot{\Phi}=\mathbf{0}$ ). Therefore, the method described into the following sections can be equally applied to both serial and parallel manipulators.

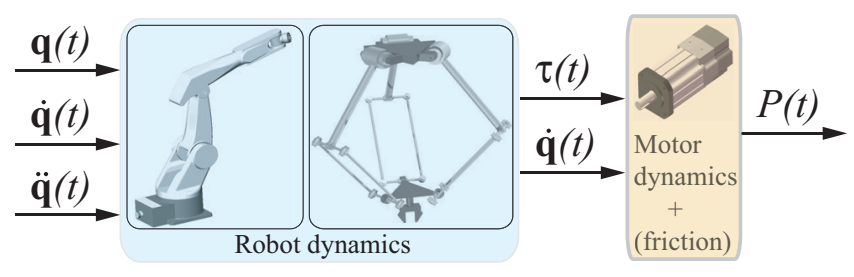

Fig. 4. Calculation of the power consumption

\section{Actuation Subsystem}

Fig. 4 shows a simplified conceptual scheme of a servosystem (position-controlled electric motor + speed reducer). The corresponding lumped-parameter model can be written as $(i=1 \ldots n)$ :

$$
\begin{aligned}
v_{a, i}(t) & =R_{a, i} i_{a, i}(t)+L_{a, i} \frac{d i_{a, i}(t)}{d t}+K_{v, i} \dot{q}_{m, i}(t) \\
I_{r, i} \ddot{q}_{m, i}(t) & =K_{T, i} i_{a, i}(t)-D_{i} \dot{q}_{m, i}(t)-\frac{\tau_{r, i}(t)}{K_{r, i}}
\end{aligned}
$$

where $i_{a, i}(t), v_{a, i}(t)$ are the supplied armature current and voltage respectively, $L_{a, i}, R_{a, i}$ are the armature electric resistance and inductance, $I_{r, i}$ is the rotor inertia, $\tau_{r, i}(t)$ is the load torque acting on the reducer output shaft, $D_{i}$ is viscous friction for the gear reducer, $K_{r, i}$ is the gear ratio, $q_{m, i}$ is the rotor angular position, $K_{v, i}$ is the back emf constant, and $K_{T, i}$ is the motor torque constant. Note that, $K_{T, i} / K_{v, i} \approx 1$ for D.C. servo motors [8]. In addition, concerning servomotors commonly used in robotic applications, the armature inductance $L_{a, i}$ can be neglected and the mechanical viscous friction coefficient is negligible with respect to the electrical friction coefficient $\left(R_{a, i}>>D_{i}\right)$.

Considering a system composed of $n$ actuators, let define the vectors $\mathbf{v}_{a}=\left[v_{a, 1} \ldots v_{a, n}\right]^{T}, \mathbf{i}_{a}=\left[i_{a, 1} \ldots i_{a, n}\right]^{T}$, $\mathbf{q}_{m}=\left[q_{m, 1} \ldots q_{m, n}\right]^{T}$, and the diagonal matrices $\mathbf{R}_{a}=\operatorname{diag}\left\{R_{a, 1} \ldots R_{a, n}\right\}, \quad \mathbf{L}_{a}=\operatorname{diag}\left\{L_{a, 1} \ldots L_{a, n}\right\}$, $\mathbf{K}_{v}=\operatorname{diag}\left\{K_{v, 1} \ldots K_{v, n}\right\}, \quad \mathbf{I}_{r}=\operatorname{diag}\left\{I_{r, 1} \ldots I_{r, n}\right\}$, $\mathbf{D}=\operatorname{diag}\left\{D_{1} \ldots D_{n}\right\}, \quad \mathbf{K}_{T}=\operatorname{diag}\left\{K_{T, 1} \ldots K_{T, n}\right\}$, $\mathbf{K}_{r}=\operatorname{diag}\left\{K_{r, 1} \ldots K_{r, 6}\right\}$. Equations 11 might be written in matrix form as:

$$
\begin{aligned}
\mathbf{v}_{a} & =\mathbf{R}_{a} \dot{\mathbf{i}}_{a}+\mathbf{L}_{a} \dot{\mathbf{i}}_{a}+\mathbf{K}_{v} \dot{\mathbf{q}}_{m} \\
\mathbf{I}_{r} \ddot{\mathbf{q}}_{m} & =\mathbf{K}_{T} \dot{\mathbf{i}}_{a}-\mathbf{D} \dot{\mathbf{q}}_{m}-\mathbf{K}_{r}^{-1} \tau_{r}
\end{aligned}
$$

and for the $n$ speed reducers

$$
\mathbf{K}_{r} \dot{\mathbf{q}}_{a}=\dot{\mathbf{q}}_{m} ; \quad \mathbf{K}_{r} \ddot{\mathbf{q}}_{a}=\ddot{\mathbf{q}}_{m}
$$

\section{Calculation of Power and Energy CONSUMPTION}

The total power supplied to the manipulator can be calculated by considering both actuator and robot dynamics (Fig. 4). In particular, the instantaneous power is given by the scalar product of the vector of actuator voltages and currents:

$$
\begin{aligned}
P & =\mathbf{i}_{a}^{T} \mathbf{v}_{a}=\mathbf{i}_{a}^{T} \mathbf{R}_{a} \mathbf{i}_{a}+\mathbf{i}_{a}^{T} \mathbf{K}_{v} \mathbf{K}_{r} \dot{\mathbf{q}}_{a}+\mathbf{i}_{a}^{T} \mathbf{L}_{a} \dot{\mathbf{i}}_{a} \\
& \approx \mathbf{i}_{a}^{T} \mathbf{R}_{a} \mathbf{i}_{a}+\dot{\mathbf{i}}_{a}^{T} \mathbf{K}_{v} \mathbf{K}_{r} \dot{\mathbf{q}}_{a}
\end{aligned}
$$

The armature current vector is found from Eqs. 12 and 13: 


$$
\mathbf{i}_{a}=\mathbf{K}_{T}^{-1} \mathbf{K}_{r}^{-1} \boldsymbol{\tau}+\mathbf{K}_{T}^{-1} \mathbf{D} \dot{\mathbf{q}}_{a}
$$

where:

$$
\begin{aligned}
\boldsymbol{\tau} & =\left(\boldsymbol{\tau}_{I}+\mathbf{G}\right)+\left({ }^{l} \boldsymbol{\tau}_{I}+{ }^{l} \boldsymbol{\tau}_{g}\right) \\
\boldsymbol{\tau}_{I} & =\left[\mathbf{M}_{1}+\mathbf{K}_{r}^{2} \mathbf{I}_{r}\right] \ddot{\mathbf{q}}_{a}+\left[\mathbf{M}_{2} \dot{\Phi}+\left(\mathbf{I}_{n} \otimes \dot{\mathbf{q}}_{a}^{T}\right) \overline{\mathbf{V}}\right] \dot{\mathbf{q}}_{a} \\
{ }^{l} \boldsymbol{\tau}_{I} & =\mathbf{J}^{T} \mathbf{h}_{I} \\
{ }^{l} \boldsymbol{\tau}_{g} & =\mathbf{J}^{T} \mathbf{h}_{g}
\end{aligned}
$$

Differently from $\tau_{r}$, the torque vector $\tau$ includes the inertial effects due to the rotor inertias $I_{r, i}$. Note that, in Eq. 16, it is convenient to split the torque contributions due to the inertia and weight of the payload ( ${ }^{l} \tau_{I}$ and ${ }^{g} \tau_{g}$, respectively) and of the manipulator ( $\tau_{I}$ and $\mathbf{G}$, respectively). By introducing Eq. 15 in Eq. 14, the following expression can be found:

$$
\begin{aligned}
P & =\boldsymbol{\tau} \mathbf{R}_{a}\left(\mathbf{K}_{r} \mathbf{K}_{T}\right)^{-2} \boldsymbol{\tau}+\boldsymbol{\tau}^{T} \mathbf{K}_{T}^{-1} \mathbf{K}_{v} \dot{\mathbf{q}}_{a}+2 \boldsymbol{\tau}^{T} \mathbf{R}_{a} \mathbf{K}_{T}^{-2} \mathbf{D} \dot{\mathbf{q}}_{a} \\
& +\dot{\mathbf{q}}_{a}^{T}\left(\mathbf{R}_{a} \mathbf{K}_{T}^{-2} \mathbf{K}_{r}^{2} \mathbf{D}^{2}+\mathbf{K}_{v} \mathbf{K}_{T}^{-1} \mathbf{K}_{r}^{2} \mathbf{D}\right) \dot{\mathbf{q}}_{a}
\end{aligned}
$$

where, as previously stated, $\mathbf{K}_{T}^{-1} \mathbf{K}_{v}=\mathbf{I}_{n}$ for D.C. servo motors commonly used in robotics [8]. Equation 18 can be denoted as:

$$
P=\tau^{T} \mathbf{R}_{1} \tau+\tau^{T} \mathbf{R}_{2} \dot{\mathbf{q}}_{a}+\dot{\mathbf{q}}_{a}^{T} \mathbf{R}_{3} \dot{\mathbf{q}}_{a}+\boldsymbol{\tau}^{T} \dot{\mathbf{q}}_{a}
$$

having defined the following matrices:

$$
\begin{aligned}
& \mathbf{R}_{1}=\mathbf{R}_{a}\left(\mathbf{K}_{r} \mathbf{K}_{T}\right)^{-2} \\
& \mathbf{R}_{2}=2 \mathbf{R}_{a} \mathbf{K}_{T}^{-2} \mathbf{D} \\
& \mathbf{R}_{3}=\mathbf{R}_{a} \mathbf{K}_{T}^{-2} \mathbf{K}_{r}^{2} \mathbf{D}^{2}+\mathbf{K}_{v} \mathbf{K}_{T}^{-1} \mathbf{K}_{r}^{2} \mathbf{D}
\end{aligned}
$$

The input power flow is then expressed by the sum of the following terms:

- $\tau^{T} \mathbf{R}_{1} \tau$ is the power dissipated by the armature resistance in the motors.

- $\tau^{T} \mathbf{R}_{2} \dot{\mathbf{q}}_{a}+\dot{\mathbf{q}}_{a}^{T} \mathbf{R}_{3} \dot{\mathbf{q}}_{a}$ corresponds to the velocity sensitive losses due to viscous friction or to coupled friction/electrical losses.

- $\tau^{T} \dot{\mathbf{q}}_{a}$ is power stored in the manipulator inertial and gravitational fields or delivered to the user (payload) [18].

In general, as said, the mechanical dissipations (viscous friction coefficients, $D_{i}$ ) are negligible with respect to the electrical dissipations (armature resistance, $R_{a, i}$ ) [19]. Assuming $\mathbf{D} \approx 0$, the power consumption can be simplified as:

$$
P=\boldsymbol{\tau}^{T} \mathbf{R}_{1} \boldsymbol{\tau}+\boldsymbol{\tau}^{T} \dot{\mathbf{q}}_{a}
$$

\section{A. Application Example}

As a simple yet significative example, suppose that the robot end effector executes cyclic pick-and-place operations for $t \in\left[0, T_{O}\right]$ whereas it is kept stationary, in a configuration $\mathbf{q}_{a}\left(T_{O}\right)$, for $t \in\left[T_{O}, T_{F}\right]$. Suppose the handled object is carried for $t \in\left[T_{A}, T_{B}\right] \subset\left[0, T_{O}\right]$. The overall energy consumption is given by:

$$
\begin{aligned}
E= & \int_{0}^{T_{F}} \boldsymbol{\tau}^{T} \mathbf{R}_{1} \boldsymbol{\tau} d t+ \\
& +\underbrace{\int_{0}^{T_{F}}\left(\boldsymbol{\tau}_{I}+\mathbf{G}\right)^{T} \dot{\mathbf{q}}_{a} d t}_{E_{I G}}+\underbrace{\int_{T_{A}}^{T_{B}}\left({ }^{l} \boldsymbol{\tau}_{I}+{ }^{l} \boldsymbol{\tau}_{g}\right)^{T} \dot{\mathbf{q}}_{a} d t}_{E_{l}}
\end{aligned}
$$

where:

- The term $E_{I G}$ represents the energy stored in the manipulator inertial/gravitational field and it is null along a closed trajectory, [18].

- The term $E_{l}$ is the energy delivered to the user and coincides with the potential energy if $\mathbf{v}\left(T_{A}\right)=\mathbf{v}\left(T_{B}\right)=\mathbf{0}$, [19].

Denoting $\Delta T_{F O}=T_{F}-T_{O}$, the energy consumption for $t \in$ $\left[T_{O}, T_{F}\right]$ is then given by:

$$
E=\mathbf{G}^{T}\left(\mathbf{q}_{a}\left(T_{O}\right)\right) \mathbf{R}_{1} \mathbf{G}\left(\mathbf{q}_{a}\left(T_{O}\right)\right) \Delta T_{F O}
$$

as long as the joints velocities are null (i.e. $\dot{\mathbf{q}}_{a}=0$ ) within the considered time frame. Therefore, the total EL for $t \in\left[0, T_{F}\right]$ is finally given by:

$$
E-E_{l}=\int_{0}^{T_{O}} \tau^{T} \mathbf{R}_{1} \tau d t+\mathbf{G}^{T}\left(\mathbf{q}_{a}\left(T_{O}\right)\right) \mathbf{R}_{1} \mathbf{G}\left(\mathbf{q}_{a}\left(T_{O}\right)\right) \Delta T_{F O}
$$

Equation 16 might then be introduced into Eq. 23 in order to better highlight the contribution of purely inertial and gravitational loads. In particular, let first discard gravitational loads. In this case, by observing that $\tau_{I}$ and ${ }^{l} \tau_{I}$ are null when the robot is stationary but increase for increasing joint velocities and accelerations (see Eq. 1 and 2), it is obvious to conclude that EL can be decreased by simply slowing down the motions. On the other hand, if only gravitational torques are considered, the EL becomes a monotonically increasing function of TET. Hence, in real conditions $(\mathbf{G} \neq \mathbf{0})$, the total EL is given by the combination of two monotonic functions with opposite trends, highlighting that there may exist a minimum for some value of TET (as shown in Fig. 2).

\section{Trajectory Time SCAling Applied to Energy CONSUMPTION MINIMIZATION}

As previously mentioned, many scheduling approaches [12], [13], assume the robots operating at their maximum achievable speed along a given path (hereafter taken as reference path). In this case, the desired position profile in the joint space is completed within $t \in\left[0, T_{O}\right], T_{O}$ being the minimum possible TET. The corresponding active joint trajectory, $\mathbf{q}_{O}(t), \dot{\mathbf{q}}_{O}(t), \ddot{\mathbf{q}}_{O}(t)$, is referred to as time-optimal trajectory [20]. In the following, all quantities referring to this time-optimal trajectory will be denoted with $O$ subscript. In industry plants, the robots are often kept stationary up to time $T_{F}$ (i.e. for the time period $\Delta T_{F O}$ ), waiting for other robots to complete their operations. The overall EL is therefore given by Eq. 23. In this situation, which happens very frequently in practice, it is possible to slow down the robot motion to decrease EL. Suppose that the aforementioned reference path is followed with a trajectory whose position profile is given by:

$$
\mathbf{q}_{s}(t)=\mathbf{q}_{o}\left(\alpha^{-1} t\right)
$$

where $\alpha \geq 1$ is denoted as scaling factor. Defining a scaled time frame as $t^{\prime}=\alpha^{-1} t, t^{\prime} \in\left[0, T_{F}\right]$, the scaled trajectory is 
given by:

$$
\begin{aligned}
\mathbf{q}_{s}(t) & =\mathbf{q}_{O}\left(t^{\prime}\right) \\
\dot{\mathbf{q}}_{s}(t) & =\frac{d \mathbf{q}_{O}\left(t^{\prime}\right)}{\alpha d t^{\prime}}=\alpha^{-1} \dot{\mathbf{q}}_{O}\left(t^{\prime}\right) \\
\ddot{\mathbf{q}}_{s}(t) & =\frac{d\left(\alpha^{-1} \mathbf{q}_{O}\left(t^{\prime}\right)\right)}{\alpha d t^{\prime}}=\alpha^{-2} \ddot{\mathbf{q}}_{O}\left(t^{\prime}\right)
\end{aligned}
$$

In the following, all quantities referring to this scaled trajectory will be denoted with $s$ subscript. In particular, the scaled object equation of motion and generalized velocity are given by:

$$
\begin{aligned}
& \mathbf{h}_{s}(t)=\alpha^{-2}\left[\begin{array}{c}
m \ddot{\mathbf{p}}_{O}\left(t^{\prime}\right) \\
\Im \dot{\omega}_{O}\left(t^{\prime}\right)+\omega_{O}\left(t^{\prime}\right) \times \Im \omega_{O}\left(t^{\prime}\right)
\end{array}\right]+\left[\begin{array}{c}
m \mathbf{g} \\
\mathbf{0}
\end{array}\right] \\
& \mathbf{v}_{S}(t)=\alpha^{-1} \mathbf{v}_{O}\left(t^{\prime}\right)
\end{aligned}
$$

The scaled generalized actuating force transformation and its time derivative (Eq. 5) are given by:

$$
\Phi_{s}(t)=\Phi_{O}\left(t^{\prime}\right) \quad \dot{\Phi}_{s}(t)=\alpha^{-1} \dot{\Phi}_{O}\left(t^{\prime}\right)
$$

In the same manner, recalling the notation defined in Eq. 16 , the scaled torque is given by:

$$
\boldsymbol{\tau}_{s}(t)=\alpha^{-2}\left(\boldsymbol{\tau}_{I O}\left(t^{\prime}\right)+{ }^{l} \boldsymbol{\tau}_{I O}\left(t^{\prime}\right)\right)+\left(\mathbf{G}_{O}\left(t^{\prime}\right)+{ }^{l} \boldsymbol{\tau}_{g O}\left(t^{\prime}\right)\right)
$$

The power input associated with the scaled trajectory is then given by:

$$
P_{s}(t)=\sum_{i=0}^{4} \alpha^{-i} p_{i}\left(t^{\prime}\right)
$$

where:

$$
\begin{aligned}
p_{4} & =\bar{\tau}_{I O}^{T}\left(t^{\prime}\right) \mathbf{R}_{1} \overline{\boldsymbol{\tau}}_{I O}\left(t^{\prime}\right) \\
p_{3} & =\bar{\tau}_{I O}^{T}\left(t^{\prime}\right)\left(\mathbf{R}_{2}-\mathbf{I}_{n}\right) \dot{\mathbf{q}}_{O}\left(t^{\prime}\right) \\
p_{2} & =2 \bar{\tau}_{I O}^{T}\left(t^{\prime}\right) \mathbf{R}_{1} \overline{\mathbf{G}}_{O}\left(\mathbf{q}_{O}\left(t^{\prime}\right)\right)+\dot{\mathbf{q}}_{O}^{T}\left(t^{\prime}\right) \mathbf{R}_{3} \dot{\mathbf{q}}_{O}\left(t^{\prime}\right) \\
p_{1} & =\overline{\mathbf{G}}_{O}^{T}\left(\mathbf{q}_{O}\left(t^{\prime}\right)\right)\left(\mathbf{R}_{2}-\mathbf{I}_{n}\right) \dot{\mathbf{q}}_{O}\left(t^{\prime}\right) \\
p_{0} & =\overline{\mathbf{G}}_{O}^{T}\left(\mathbf{q}_{O}\left(t^{\prime}\right)\right) \mathbf{R}_{1} \overline{\mathbf{G}}_{O}\left(\mathbf{q}_{O}\left(t^{\prime}\right)\right) \\
\bar{\tau}_{I O} & =\tau_{I O}+{ }^{l} \tau_{I O} \\
\overline{\mathbf{G}}_{O} & =\mathbf{G}_{O}+{ }^{l} \boldsymbol{\tau}_{g O}
\end{aligned}
$$

Supposing the overall energy input along the reference path as given, then the EL concerning the scaled trajectory can computed as a polynomial function in the scaling factor $\alpha$ :

$$
\begin{aligned}
E_{S}(t) & =\int_{0}^{T_{F}} P_{S}(t) d t=\int_{0}^{T_{O}} P_{S}\left(t^{\prime}\right) \alpha d t^{\prime} \\
& =\sum_{i=0}^{4} \alpha^{1-i} \int_{0}^{T_{O}} p_{i}\left(t^{\prime}\right) d t^{\prime}=T_{O} \sum_{i=0}^{4} \alpha^{1-i} \bar{p}_{i}\left(t^{\prime}\right)
\end{aligned}
$$

where $\bar{p}_{i}\left(t^{\prime}\right)=T_{O}^{-1} \int_{0}^{T_{O}} p_{i}\left(t^{\prime}\right) d t^{\prime}, i=1 \ldots 4$, are mean powers due to the various contributions highlighted in Eq. 29. Note the change of integration variable in Eq. 31 as $t=\alpha t^{\prime}$.

The efficiency of the proposed scaling method can be computed by means of the EL ratio, defined as

$$
\eta_{E L}=\frac{E_{S}-E_{l}}{E_{O}-E_{l}}
$$

where $E_{s}$ and $E_{O}$ are the EL related to the scaled and timeoptimal trajectory respectively. In particular, the energy $E_{O}$ is simply given by Eq. 31, setting $\alpha=1$ whereas $E_{l}$, being the potential energy delivered to the user, remains unaltered after time-scaling. Considering once again a robot performing a pick-and-place operation for $t \in\left[0, T_{O}\right]$ and kept stationary for $t \in\left[T_{O}, T_{F}\right]$, the efficiency ratio may be computed as

$$
\widetilde{\eta}_{E L}=\frac{\int_{0}^{T_{F}} \boldsymbol{\tau}_{O}^{T} \mathbf{R}_{1} \boldsymbol{\tau}_{s} d t}{\int_{0}^{T_{O}} \boldsymbol{\tau}_{O}^{T} \mathbf{R}_{1} \tau_{O} d t+\mathbf{G}^{T}\left(\mathbf{q}_{O}\left(T_{O}\right)\right) \mathbf{R}_{1} \mathbf{G}\left(\mathbf{q}_{O}\left(T_{O}\right)\right) \Delta T_{F O}}
$$

where the mechanical viscous friction has been reasonably neglected. Note that Eq. 33 simplifies whenever the robot actuators are equal, as it generally happens in parallel manipulators. In such a case, the contribution of the matrix $\mathbf{R}_{1}$ disappears, the efficiency ratio becoming independent of the actuator system parameters (i.e. stator resistance $R_{a, i}$, equivalent torque constant $K_{T, i}$, gear ratio $K_{r, i}$ ).

\section{Simulation RESUlts}

A case study is simulated in order to assess the validity of the proposed approach. The PKM depicted in Fig. 1(b) performs cyclic pick-and-place operations, following a closed loop path in the 3D cartesian space. The PKM electromechanical parameters are taken from [21], [22]. The handled object is initially still, it is then grabbed to its position and finally delivered at null velocity in a different position. A reference joint space trajectory $\mathbf{q}_{O}(t), \dot{\mathbf{q}}_{O}(t), \ddot{\mathbf{q}}_{O}(t)$ is found by standard inverse kinematics techniques [19]. This same trajectory is regarded as time-optimal even if the actuators limits are not reached. The simulated angular position profiles of the three rotors are reported in Fig.5. The robot is assumed stationary until $T_{F}=1.5 \mathrm{~s}$ if the desired operation is completed for $T<T_{F}$. Figure 5 also shows the effects of the trajectory time-scaling by means of a constant scaling factor, $\alpha=1.5$, as described in Eq.24. A comparison between the original trajectories (continuous lines) and the scaled ones (dashed lines) trivially shows that the profile is constantly stretched in time axis direction. With reference to the same pick-and-place task, Fig.6 reports, as an example, the total simulated torque supplied by rotor 2 . The comparison between the torque concerning the original trajectory (continuous lines, $\alpha=1$ ) with the scaled one (dashed lines,

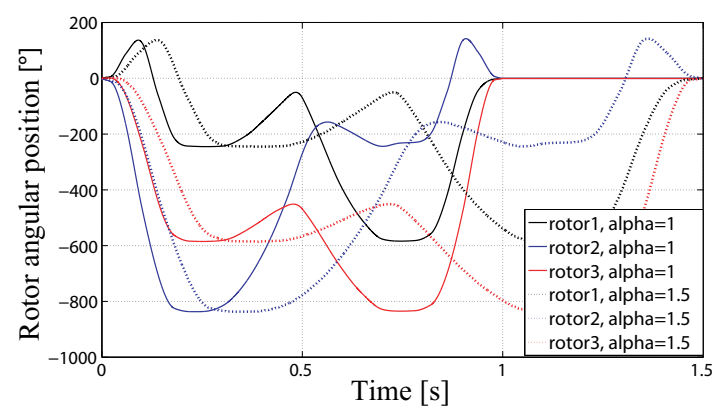

Fig. 5. Time-optimal joint space trajectory, $\alpha=1$ and scaled joint space trajectory, $\alpha=1.5$. Simulation results. 


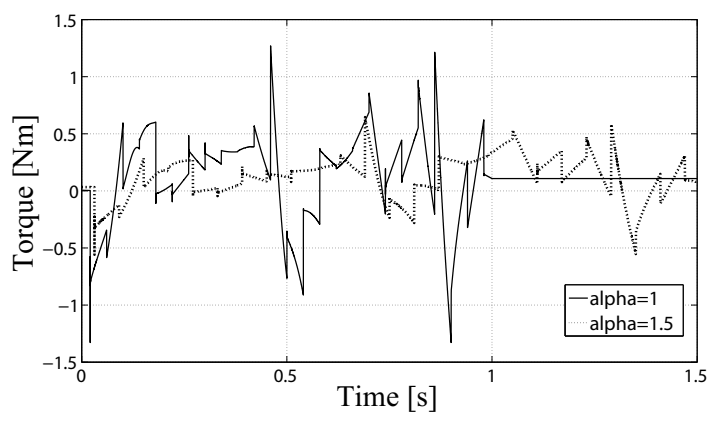

Fig. 6. Joint torque corresponding to the time-optimal trajectory, $\alpha=1$, and to the scaled trajectory, $\alpha=1.5$. Simulation results.

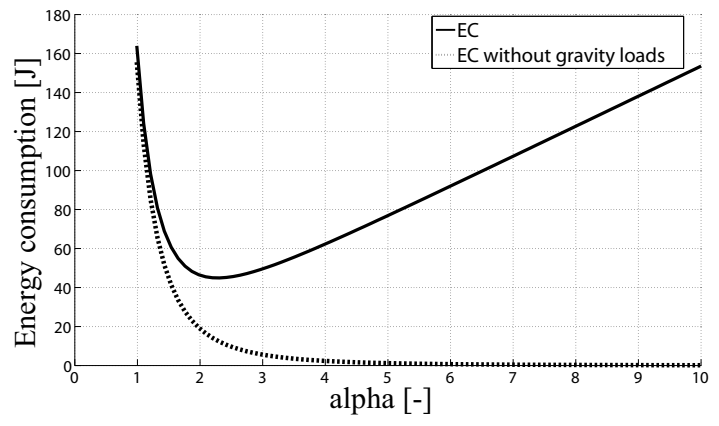

Fig. 7. Energy consumption as a function of scaling factor $\alpha$ in case of complete electromechanical model and absence of gravity. Simulation results.

$\alpha=1.5$ ) shows that the effect of time scaling is not as readily predictable as it was concerning the joint positions. Finally, Fig.7 reports the simulated EL as a function of the scaling factor $\alpha$ (continuous line). In particular, it is evident that the EL presents a local minimum achieved for $\alpha=2.2$. In addition, the same figure reports the EL profile in case of absence of gravitational load (dotted line) highlighting that, in such a case, energy consumption is a monotonic function decreasing at increasing scaling factor (i.e. slowing down the operation). The efficiency $\eta_{E L}$ of the scaling procedure has been evaluated, resulting in an EL decrease of $12.1 \%$ (for $\alpha=2.2$ ) with respect to the reference trajectory. Nonetheless, the overall efficiency of the proposed method should be evaluated on multi-robot operations [9], [11], where existing scheduling approaches would be capable of guaranteeing energy saving with virtually no negative effects on the overall system productivity.

\section{CONCLUSIONS}

An engineering method for energy consumption optimization of robotic systems has been presented. The method is applicable to both serial and parallel manipulators whose dynamic models are known. Differently from other energy minimization routines, the proposed strategy does not rely on either equipment replacement, plant modification or path replanning. In fact, starting from given manipulator electromechanical parameters and pre-scheduled trajectories performed at maximum speed (i.e. compatible with actuators limitations), an energy-optimal trajectory is determined by means of time-scaling (i.e. simply slowing down the operation and reducing the manipulator idle times). The approach has been tested on a common parallel robot performing a cyclic pick-and-place operation. The results allow the operator to parameterize and adjust the manipulator operation in order to reduce the energy consumptions, when allowed by other scheduling or manufacturing constraints. Future work includes the development of on-line programming algorithms.

\section{REFERENCES}

[1] P. Menanteau, D. Finon, and M. Lamy. Prices versus quantities: choosing policies for promoting the development of renewable energy. Energy Policy, 31:799-812, 2003.

[2] F. Roos, H. Johansson, and J. Wikander. Optimal selection of motor and gearhead in mechatronic applications. Mechatronics, 16:63-72, 2006.

[3] T. Izumi, H. Zhou, and Z. Li. Optimal design of gear ratios and offset for energy conservation of an articulated manipulator. IEEE Tran. on Automation Science and Engineering, 6(3):551-557, 2006.

[4] N. Meyer and J. Angeles. Minimization of power losses in cooperating manipulators. American Control Conference, pages 3044 -3049, 1991.

[5] R. Saidur. A review on electrical motors energy use and energy savings. Renew. Sust. Energ. Rev., 14:877-898, 2010.

[6] R. Visinka. Ch. 2 - Energy Efficient Three-Phase AC Motor Drives for Appliance and Industrial Applications. Green Electronics, Ed. Goldberg and Middleton, 2000.

[7] H. Diken. Energy efficient sinusoidal path planning of robot manipulators. Mech. Mach. Theory, 29(6):785-792, 1994.

[8] J.S. Park. Motion profile planning of repetitive point-to-point control for maximum energy conversion under acceleration conditions. Mechatronics, 6 (6):649-663, 1996.

[9] A. Vergnano et al. Embedding detailed robot energy optimization into high-level scheduling. IEEE Case, Int. Conf. on Automation Science and Engineering, pages $386-392,2010$.

[10] G. Hirzinger et. al. DLR's torque-controlled light weight robot III Are we reaching the technological limits now? Proc. IEEE ICRA Int. Conf. on Robotics and Automation, 2:1710-1716, 2002.

[11] B. Lennartson et al. Hybrid cost automata applied to energy optimization. Proc. Int. Conf. on Hybrid Systems: Computation and Control, 2010.

[12] S. Panke, O. Stursberg, and S. Engell. Optimization of timed automata models using mixed integer programming. Formal Modelling and Analysis of Timed Systems, Springer Berlin, pages 73-87, 2004.

[13] A.Kobetski and M. Fabian. Time-optimal coordination of flexible manufacturing systems using deterministic finite automata and mixed integer linear programming. Discrete Event Dyn S, 19(3):287-315, 2009

[14] S. B. Moon and S. Ahmad. Time-optimal trajectories for cooperative multi-manipulator systems. IEEE Tran. on Systems, Man, and Cybernetics-part B, 27(2):343353, 1997.

[15] J. Craig. Introduction to Robotics: Mechanics and Control (3rd Edition). Addison-Wesley Publishing Company, Inc., 2005.

[16] F. L. Lewis, C. T. Abdallah, and D. M. Dawson. Control of Robot Manipulators: Theory and Practice. Marcel Dekker, Inc. New York, 2004

[17] F. C. Park, J. Choi, and S. R. Ploen. Symbolic formulation of closed chain dynamics in independent coordinates. Mechanism and Machine Theory, 34(5):731 - 751, 1999.

[18] C. Batlle et al. Ch. 3 - Modeling and Control of Complex Physical Systems The Port-Hamiltonian Approach. Ed. Duindam, Macchelli, Stramigioli, Bruyninckxn. Springer, London, 2009.

[19] L. Sciavicco, B. Siciliano, L. Villani, and G.Oriolo. Robotics: Modelling, Planning and Control. Springer, London, 2009.

[20] J.E. Bobrow, S. Dubowsky, and J.S. Gibson. Time-optimal control of robotic manipulators along specified paths. International Journal of Robotics Research, 4(3):3-17, 1985.

[21] P. Guglielmetti. Model based control of fast parallel robots: a global approach in operational space. $\mathrm{PhD}$ thesis, Ecole Polytechnique federale de Lausanne EPFL, Lausanne, CH, 1994.

[22] Y. Li and G.M. Bone. Are parallel manipulators more energy efficient? IEEE International Symposium on Computational Intelligence in Robotics and Automation, Bannf, CDN, page 4146, 2001. 